\title{
PD49 - Induced sputum versus exhaled nitric oxide for the evaluation of airway inflammation in allergic pediatric asthma patients treated with omalizumab
}

\author{
Mireia Vilella, Montserrat Bosque, Juan Trujillo*, Xavier Domingo, Elisabet Guijarro, Òscar Asensio, Laura Valdesoiro, \\ Helena Larramona, Alba Maestro, Christian Domingo
}

From 3rd Pediatric Allergy and Asthma Meeting (PAAM)

Athens, Greece. 17-19 October 2013

\section{Purpose}

To determine the inflammatory changes in the airways of allergic pediatric asthma patients treated with omalizumab, measured by the percentage of eosinophil in induced sputum and exhaled nitric oxide.

\section{Methods}

From 2006 to 2012, 31 patients with asthma were treated with omalizumab (15 male -51.6\%; 16 females -48,4\%). Age ranged between $6-18$ years. The dose of omalizumab was calculated according to the dosing table of the company. Omalizumab was administered subcutaneously in the hospital.

\section{Protocol}

Baseline analysis from every patient: Total IgE concentration, specific IgE against the relevant allergen, the percentage of eosinophils in a smear of induced sputum and the exhaled fraction of nitric oxide (NO). Induced sputum and NO were measured at the end of follow-up. Data are shown as mean (SEM). A Student $\mathrm{t}$-test for paired data was used for comparison.

\section{Results}

Follow-up of patients was not uniform, ranging from 2-6 years. Total IgE concentration at entry: 668.89 (117.79) $\mathrm{IU} / \mathrm{mL}$; Specific IgE against the major antigen at entry: 42.15 (7.32) IU/mL; ( 22 house dust mite; 7 alternaria; 2 cladosporium). Initial and end induced sputum: 6.26
$(2.03) \%$ vs $2.47(0.36) \% ;(\mathrm{p}<0.05)$. Initial and end NO values: 19.04 (1.98) ppb vs 18.10 (2.11) ppb ( $\mathrm{p}=\mathrm{NS})$. Three patients were excluded from the evaluation due to exagerated values in final NO measurement that preceded a severe exacerbation.

\section{Conclusions}

Omalizumab allowed a statistically significant decrease in the percentage of eosinophils in induced sputum of this cohort of patients. Although very sensible, NO is a less reproducible and thus less reliable method to evaluate chronic airway inflammation in a pediatric allergic population with uncontrolled severe asthma. Induced sputum seems to be a better method to monitor chronic inflammation and thus the response to chronic omalizumab treatment while NO measurement would be more useful to monitor acute events preceding exacerbations.

Published: 28 February 2014

doi:10.1186/2045-7022-4-S1-P49

Cite this article as: Vilella et al:: PD49 - Induced sputum versus exhaled nitric oxide for the evaluation of airway inflammation in allergic pediatric asthma patients treated with omalizumab. Clinical and Translational Allergy 2014 4(Suppl 1):P49.

\footnotetext{
Corporació Sanitària Parc Taulí, Sabadell, Barcelona, Spain
} 